\title{
Reflections on HLA Epitope-Based Matching for Transplantation
}

\author{
Rene J. Duquesnoy* \\ University of Pittsburgh Medical Center, Pittsburgh, PA, USA
}

HLA antibodies are primary causes of transplant rejection; they recognize epitopes that can be structurally defined by eplets. There are many reviews about HLA epitope-based matching in transplantation. This article describes some personal reflections about epitopes including a historical perspective of HLA typing at the antigen and allele levels, the repertoires of antibody-verified HLA epitopes, the use of HLAMatchmaker in determining the specificities of antibodies tested in different assays, and, finally, possible strategies to control HLA antibody responses.

Keywords: HLA, epitope, eplet, antibody, mismatch acceptability

\section{INTRODUCTION}

OPEN ACCESS

Edited by:

Narinder K. Mehra

All India Institute of Medical

Sciences, India

Reviewed by:

Myra Coppage,

University of Rochester

Medical Center, USA

Eric Spierings,

Utrecht University, Netherlands

*Correspondence:

Rene J. Duquesnoy

duquesnoyr@upmc.edu

Specialty section:

This article was submitted to Alloimmunity and Transplantation,

a section of the journal

Frontiers in Immunology

Received: 22 August 2016

Accepted: 18 October 2016

Published: 28 November 2016

Citation:

Duquesnoy RJ (2016) Reflections on HLA Epitope-Based

Matching for Transplantation.

Front. Immunol. 7:469.

doi: 10.3389/fimmu.2016.00469
It is now universally accepted that HLA matching affects transplant outcome and that HLA antibodies are primary causes of transplant rejection. HLA antibodies recognize epitopes, which can be structurally defined by eplets, i.e., small configurations of polymorphic amino acid configurations on the HLA molecular surface. This article addresses the concept that HLA matching can be determined at the epitope level. It is not intended as an extensive review of the literature; the www.HLAMatchmaker.net website has numerous epitope-related publications, and there are several review articles (1-4). Rather, this paper offers some recent reflections about the role of HLA epitopes in histocompatibility.

\section{EPITOPES AND A HISTORICAL PERSPECTIVE OF SEROLOGICAL HLA TYPING}

HLA emerged from observations by a few investigators including Rose Payne, Jon van Rood, and Jean Dausset who during the early 1960s studied sera with leukocyte antibodies in patients with non-hemolytic transfusion reactions and in women after pregnancies (5). Most reactivity patterns with leukocyte panels were uninterpretable, until international HLA workshops were organized whereby collaborating laboratories adapted the so-called microdrop complement-dependent lymphocytotoxicity technique developed by Terasaki and McClelland (6). Sera could be grouped into non-overlapping clusters with highly correlated reactivity patterns, and this permitted assignments of specificities such as HLA-A1, A2, B5, and B7. Such clusters served as reference standards for serological HLA typing reagents. Later on, subclusters of sera identified the so-called splits such as A10 was split into A25 and A26, and B16 was split into B38 and B39. Continued workshop efforts led to a set of HLA-class I specificities also called antigens that could be identified serologically with the complement-dependent lymphocytotoxicity technique. Most HLA antigens were defined with the so-called monospecific sera, but many others could be only identified from reactivity patterns of selected sera on typing trays.

Yunis and Amos first proposed the HLA-D locus from cellular assays based on mixed lymphocyte reactivity (7). Specific Dw determinants were later identified with HLA-D homozygous typing 
cells and primed alloreactive lymphocytes. Certain sera had antibodies with blocking effects on lymphocyte reactivity and with complement-dependent cytotoxicity assays using B-cells, and it was possible to identify serum clusters specific for serologically defined Dw related or DR antigens now referred to as DR1, DR2, DR3, etc. (8). Subclusters of sera also identified "splits" such as DR11 and DR12 of DR5.

HLA workshop studies during the 1980s identified clusters of sera specific for the DRB3/4/5-encoded antigens DR51, DR52, and DR53 and the DQB antigens DQ1, DQ2, DQ3, and DQ4 and again subclusters of sera demonstrated "splits" such as the DQ5 and DQ6 splits of DQ1.

As noted above, serological typing was primarily based on reactivities of specific antisera with antigens. Since it is now recognized that HLA antibodies are specific for epitopes rather than antigens, it seems obvious that HLA-typing sera must recognize distinct epitopes uniquely present on serologically defined antigens. The HLAMatchmaker analysis has shown that many HLA antigens detected by the so-called monospecific or duospecific sera have unique eplets, and almost all of them are recorded in the International HLA Epitope Registry (http://www.epregistry.com.br) as experimentally verified with informative antibodies (Table 1). In other words, antiA1 antibodies actually recognize the 163RG eplet, which is only found on $\mathrm{A} 1$, anti-B7 antibodies are specific for $177 \mathrm{DK}$ uniquely present on $\mathrm{B} 7$, and anti-Cw1 antibodies recognize an epitope defined by $6 \mathrm{~K}$. Several serological splits can be explained by eplets paired with other residue configurations. For instance, A10 corresponds to 149TAH, whereas the A25 and A26 splits represent 149TAH $+80 \mathrm{I}$ and $149 \mathrm{TAH}+80 \mathrm{~N}$, respectively. Similarly, the B38 and B39 splits of B16 are defined by antibodies specific for epitopes defined by the $158 \mathrm{~T}+80 \mathrm{I}$ and $158 \mathrm{~T}+80 \mathrm{~N}$ pairs, respectively. Also, Table 1 illustrates that most serologically defined DR and DQ antigens have uniquely distinct eplets. The cellularly defined DP specificities [originally called SB (9)] do not have unique eplets, and this explains why they cannot be readily determined serologically with monospecific sera.

The information in Table $\mathbf{1}$ is based on molecular structure and amino acid sequence information of HLA antigens which did not emerge until after the late 1980s. Before that, the specificities of serum clusters in the early workshops could only be designated with an arbitrary notation system of serologically defined antigens, although we can now readily see that they reflect the recognition of distinct epitopes. Accordingly, the HLA antigenmatching effect on transplant outcome can be reinterpreted as actually demonstrating the influence of matching for epitopes, albeit limited numbers were considered in these association analyses.

\section{EPITOPES AND SEROLOGICALLY CROSS-REACTING GROUPS}

Many sera with HLA antibodies exhibit complex reactivity patterns that prohibit their use in serological typing. Early studies identified sera that reacted with the so-called crossreacting groups (CREGs) of HLA antigens; the A2-CREG and B7-CREG are common examples $(10,11)$. Each CREG has the so-called public determinants shared between certain

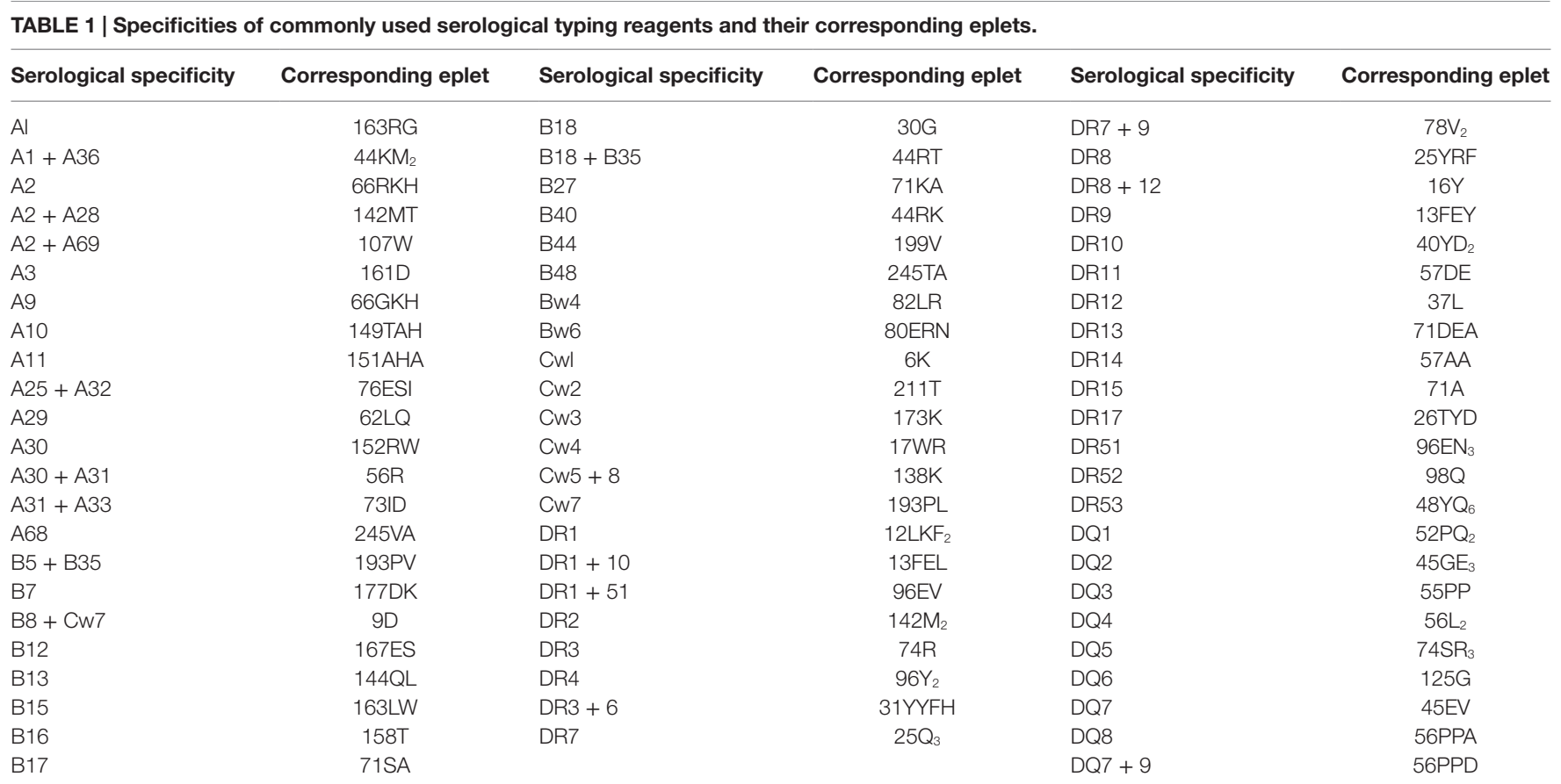

Eplet descriptions are according to HLAMatchmaker (www.epitopes.net). The number represents the sequence location of one of the polymorphic residues annotated with standard single letters. Some eplets have subscripted numbers indicating additional residue configurations in other locations. 
groups of antigens and so-called private determinants limited to a given serologically defined antigen within the CREG (12). Many public determinants correspond to structurally defined epitopes. As an example, the A2-CREG, which includes A2, A23, A24, A68, A69, and B17, has several epitopes corresponding to public determinants including the $127 \mathrm{~K}$ eplet on $\mathrm{A} 2+\mathrm{A} 23+\mathrm{A} 24+\mathrm{A} 68+\mathrm{A} 69,144 \mathrm{TKH}$ on $\mathrm{A} 2+\mathrm{A} 68+\mathrm{A} 69$ $107 \mathrm{~W}$ on $\mathrm{A} 2+\mathrm{A} 69$, and $62 \mathrm{GE}$ on $\mathrm{A} 2+\mathrm{B} 17$, and all of them have been antibody verified. It has become evident that all HLA class I antigens have public determinants or shared epitopes that can be recognized by antibodies.

Although CREG matching has been applied to the identification of compatible platelet donors for refractory alloimmunized thrombocytopenic patients (13), no beneficial effect could be convincingly demonstrated on transplant outcome largely because these studies did not consider individual public epitopes. Most highly sensitized patients have antibodies against public epitopes that can now be defined structurally.

\section{EPITOPE-BASED MATCHING REQUIRES DNA-BASED HLA TYPING AT THE ALLELE LEVEL}

Serological HLA typing had always accuracy problems because of the lack of reagents and technical limitations. After elucidation of the HLA molecular structure and nucleic acid sequencing of HLA antigens during the late 1980s, the application of DNA-based technologies permitted accurate HLA antigen typing results. Very soon, many antigens were identified with amino acid differences, and this led to assignments of alleles to be annotated with a colon (:) followed by two and later on three digits after more than 100 alleles corresponding to the 2-digit antigen had been identified (Examples are $A^{\star} 02: 01$ and $\left.A^{\star} 02: 101\right)$. Certain amino acid differences affect the expression of eplets. For instance, $A^{\star} 24: 02$ has the antibody-verified 166DG eplet shared with $A^{\star} 01: 01, A^{\star} 23: 01, A^{\star} 80: 01$, and $B^{\star} 15: 12$, whereas $A^{\star} 24: 03$ has $166 \mathrm{EW}$ shared with $A^{\star} 02: 01$, $A^{\star} 03: 01$, etc. This example illustrates the difficulty of matching at the antigen level, in this case A24 if the patient has antibodies against $166 \mathrm{DG}$ or $166 \mathrm{EW}$. It is now recognized that HLA compatibility is better determined at the allele than at the antigen level (14).

There are thousands of HLA alleles and the question arises which ones should be typed for in the clinical transplant setting. One might focus on alleles present in the patient and donor population of a given transplant program. Although rare alleles might be excluded it is now apparent that because of the increasing racial and ethnic heterogeneity of most populations such alleles occur more frequently as mismatches. Tissue typing techniques are moving forward very fast in the clinical setting, and each HLA allele has precise information about its eplet repertoire. The degree of eplet mismatching of a donor allele depends on the HLA phenotype of the recipient, which has its own repertoire of self-eplets. Such determinations can be readily made with specifically designed computer programs such as HLAMatchmaker.

\section{ANTIBODY REACTIVITY WITH HLA EPITOPES}

Epitope-based HLA compatibility determination requires a basic understanding of how antibodies interact with epitopes. Antibody reactivity testing with HLA panels can be done with different techniques from immunoglobulin and complement component $\mathrm{C1q}$ binding to isolated HLA molecules attached to microbeads to flow cytometric binding on lymphocytes and complement-dependent lymphocytotoxicity. These methods may give different results which make clinical relevance assessments difficult. Epitope specificity analyses of antibodies may clarify some of these issues.

Antibody binding to protein epitopes occurs through six complementarity-determining region (CDR) loops, three of them $\mathrm{H} 1, \mathrm{H} 2$, and $\mathrm{H} 3$ are on the immunoglobulin heavy chain and L1, L2, and L3 are on the light chain. Each loop interacts with a small set of amino acid residues in the so-called structural epitope and CDR-H3 which binds to the so-called functional epitope (or hot spot) in a central location has a dominant role in determining antibody specificity. Eplets are considered to be equivalent to functional epitopes. Figure 1 shows models of eplets in three different locations on class I molecules and in context with corresponding structural epitopes and hypothetical contact sites for the CDRs of antibody. Two eplets are readily antibody accessible on the upper domains (Figures 1A,B), but antibodies to eplets on the membrane-proximal domain (Figure 1C) might interact with only solubilized but not with lymphocyte membrane-bound HLA molecules because these epitopes may not be readily antibody accessible. Would such epitopes be clinically significant?

Antibodies through their CDR-H3 recognize specific eplets centrally located in the structural epitope. Other eplets in the same sequence location but with different residue compositions are generally non-reactive, but there can be exceptions if the eplet has a high degree of residue homology with the eplet that had induced the antibody. As an example, the 82LR eplet, which describes the well-known Bw4 epitope, has two closely nearby residues $80 \mathrm{I}$ and $80 \mathrm{~T}$. Many $80 \mathrm{I}+82 \mathrm{LR}$-induced antibodies recognize also $80 \mathrm{~T}+82 \mathrm{LR}$ and binding strengths can vary from high to low. This is an example of the so-called Landsteiner type of serological cross-reactivity, whereby different but structurally related epitopes react with the same antibody. It should be noted that certain 80I $+82 \mathrm{LR}$-induced antibodies never react with $80 \mathrm{~T}+82 \mathrm{LR}$; they can be designated as specific for only $80 \mathrm{I}$. This epitope-based analysis seems helpful in the assessment of the mismatch acceptability of 82LR (or Bw4) when 80T + 82LR-carrying alleles have no or very low reactivity with patient's serum.

As illustrated in Figure 1, the structural epitope has other amino acid configurations that interact with the remaining CDRs of antibody. As described in many immunology textbooks, an important consideration is the so-called affinity maturation process during the antibody response, whereby DNA regions corresponding to CDRs undergo mutations which increase antibody affinity with the structural epitope. As an example, let us just consider for Figure 1A one CDR-loop L2, which as a result of affinity maturation, has increased binding with a given amino acid 

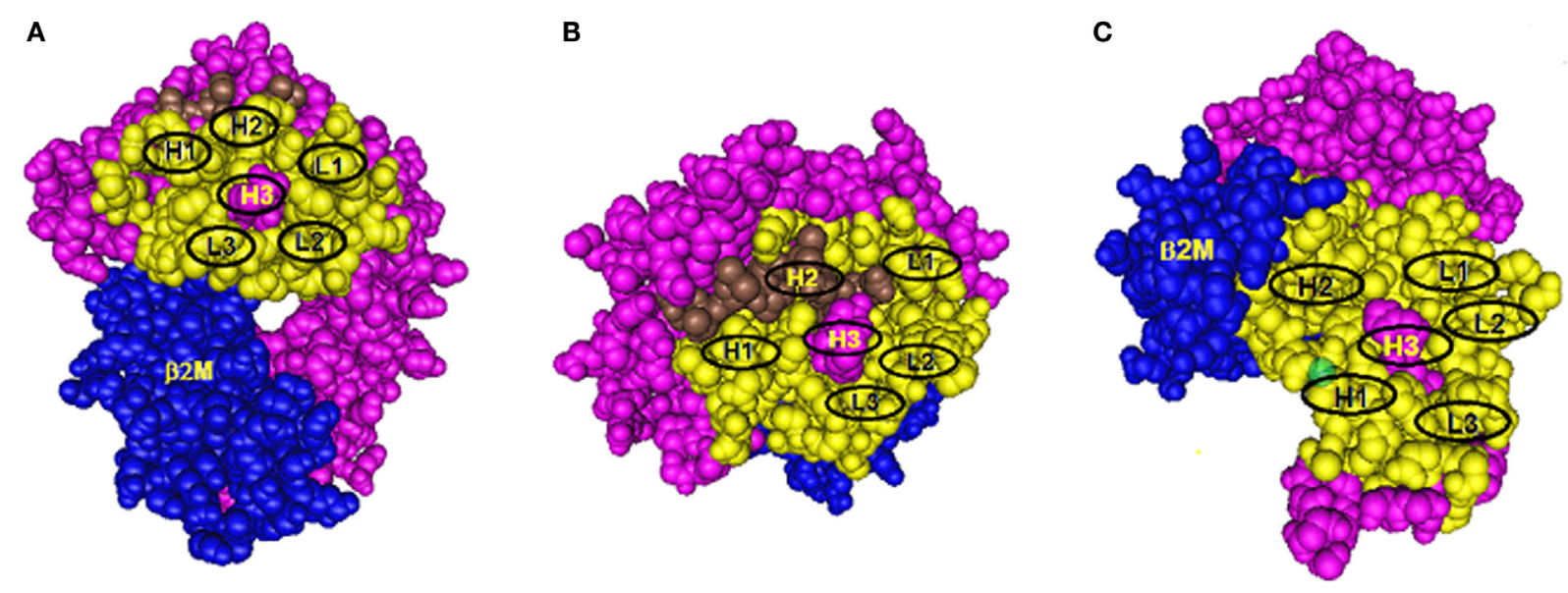

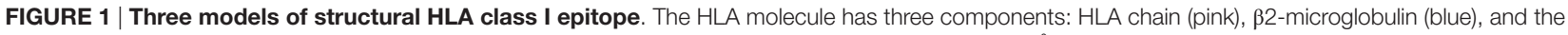
bound peptide (green). The centrally located eplet (in pink) interacts with CDR-H3. Residues within a 15- $\AA$ radius are colored yellow and include configurations (in oval circles) that make contact with other CDRs on heavy chain ( $\mathrm{H} 1$ and $H 2)$ and light chain $(\mathrm{L} 1, \mathrm{~L} 2$, and L3). (A-C) reflect three different epitope locations on the molecule.

configuration in the structural epitope of the immunizing allele. Two possible configurations can be considered. One comprises only monomorphic residues shared between all alleles in the panel, and the epitope recognized by antibody corresponds solely to an eplet. Second, this configuration has polymorphic residues and only alleles that share such residues with the immunizing allele are antibody reactive. Such residues represent critical contact sites for antibody. Accordingly, these epitopes can be defined by eplets paired with distinct residue configurations (including eplets) in other sequence locations within a 15 - $\AA$ radius, a presumed dimension of a structural epitope. As an example, there are antibodies specific for 82LR paired with 145RA; this epitope is present on all 82LR-carrying alleles except A25 and B13 that have $82 \mathrm{LR}+145 \mathrm{RT}$ and $82 \mathrm{LR}+145 \mathrm{LA}$, respectively. It should be noted that the critical contact residue configurations are almost uniformly present on at least one allele of the antibody producer, and this suggests an autoimmune component of the antibody response to a mismatched eplet.

As illustrated in Figure 1B, some CDRs can make contact with peptides bound to the groove, and it is possible that peptides serve as critical contact sites for a CDR such as H2. Indeed, it has been reported that certain HLA antibodies are peptide dependent (15-17).

For many protein antigen-antibody complexes, there is a certain level of permissiveness for residue substitutions in critical contact areas, and this applies also for HLA epitopes. Certain residue substitutions in the structural epitope have a moderate effect on an allele's reactivity with antibody, whereas others are inhibitory to the level of weak reactivity or non-reactivity of specific eplet-carrying alleles. The latter might be considered as epitope-based acceptable mismatches.

The same rules apply to epitope descriptions of class II alleles which more often solely correspond to eplets. Only few DRB epitopes correspond to eplet pairs, and this might be due to monomorphic residue nature of the DRA chain. Several immunogenic DRB1 eplets are also on alleles encoded by DRB3, DRB4, and/or DRB5. Each of them has distinct antibody-verified eplets, and it seems that epitope matching for DRB3/4/5 affects the class II antibody response.

DQ and DP encode for heterodimers of A and B chains which are both polymorphic and have distinct eplets many of which have been antibody verified. DQ-specific antibodies appear most prevalent among antibodies induced by class II mismatches. Such antibodies are specific for eplets on DQA and DQB chains, and there is emerging evidence that some DQ epitopes are defined by eplet pairs involving both chains $(18,19)$. This suggests that epitope-based matching should consider the DQ heterodimer rather than the individual chains alone. DP mismatching involves generally fewer epitopes on DPB and especially on DPA; immunogenic eplets, such as $84 \mathrm{DEAV}$ and 55DE, are present on large groups of DPB alleles.

\section{REPERTOIRES OF ANTIBODY-VERIFIED HLA EPITOPES}

Eplets are small configurations of amino acid residues that play dominant roles in HLA epitopes reactive with antibodies. Such configurations are theoretical considerations based on residue differences in polymorphic sequence locations, but we must raise the question how many of them are actually recognized by specific antibodies. One would expect the clinical relevance of epitope-based matching to apply only to epitopes that have been experimentally verified with informative antibodies. The HLA Epitope Registry has a list of antibody-verified epitopes recorded this far for each locus, but the repertoire is still incomplete. Very recently, the website includes a downloadable PDF file "EpiPedia of HLA" that describes the antibody verifications of HLA epitopes in detail. With the help of participating HLA laboratories that might have interesting serum antibody reactivity patterns, we will continue our analyses to identify new epitopes. The HLAMatchmaker website www.epitopes.net (formerly www.HLAMatchmaker.net) has now a downloadable 
Excel document "Five Maps of HLA Epitopia," which describes the sequence locations of antibody-verified eplets and polymorphic residues as potential candidates defining additional epitopes. These maps can be used in navigating the continents of HLA Epitopia while searching for newly antibody-defined epitopes (20).

\section{TECHNIQUE-DEPENDENT EPITOPE SPECIFICITIES OF HLA ANTIBODIES}

The use of C1q-binding and complement-dependent lymphocytotoxicity assays has added other dimensions of the complexity of the antibody response to a mismatch. These tests are based on sequential events following the formation of the antibodyepitope complex. $\mathrm{Clq}$ binding requires a conformational change in the antibody molecule, thereby exposing the $\mathrm{Clq}$ receptor on the Fc part, and complement-dependent cytotoxicity is initiated by the activation of antibody-bound C1qrs complex as the first step of the classical complement pathway. Both processes require free energy released during antibody-epitope complex formation, and the amount depends on the binding strength between all CDRs with the structural epitope. Some antibodies react only with the immunizing epitope in Ig-binding assays; this means that the amount of free energy release is insufficient for the activation of complement-dependent mechanisms that contribute to inflammatory responses $(21,22)$. A given antibody can react with the immunizing eplet and certain eplet-sharing alleles in the panel in all three assays. Other eplet-carrying alleles react only in Ig-binding assays, because they lack certain critical residues in the corresponding structural epitopes required for strong binding with antibody required for the initiation of the inflammatory process. Are such alleles acceptable or unacceptable mismatches?

\section{PRETRANSPLANT SERUM TESTING FOR EPITOPE SPECIFICITIES OF HLA ANTIBODIES}

The above interpretations about antibody reactivity and epitope specificity are more readily made with monospecific sera. However, most sera from sensitized patients have mixtures of antibodies, and although the reactivity patterns are generally limited to a few specificities, there are additional features that can make epitope-based interpretations often quite challenging. They include unexpected reactivities of certain panel alleles due to "natural" antibodies or non-specific blocking factors including a prozone effect; sera may also have competing antibodies with different characteristics including Ig subtypes. Many sera from highly sensitized patients have antibodies reacting with high-frequency (i.e., $>80 \%$ ) epitopes, which make detections of antibodies against lower frequency epitopes more difficult unless these antibodies are separated through absorption-elution studies with selected alleles.

Technique dependencies of serum reactivity may also affect the interpretation of epitope specificities, especially for highly sensitized patients who have several antibody populations in different concentrations and affinities that affect their reactivity with
HLA panels. Again, absorption-elution studies with selected alleles might dissect these serum reactivity patterns, so that an epitope analysis can be more readily done.

TheHLAMatchmakerwebsitehasthreedownloadableantibody analysis programs in Excel format: HLA-ABC, HLA-DRDQDP, and MICA. The latest 02 versions focus on antibody-verified epitopes recorded so far in the HLA Epitope Registry. All of them correspond to single eplets or eplets paired with other residue configurations uniquely shared by a group of antibody-reactive alleles. The antibody analysis programs also include "other" theoretical eplets, which might become experimentally verified if informative antibodies are identified. The HLAMatchmaker website has a downloadable instruction manual for the epitope analysis of HLA antibodies tested in assays with single alleles.

The 02 versions require entering of the HLA information of the panel, the MFI values of each allele and the allelic HLA type of the patient and preferably the immunizer (e.g., a previous transplant or in case of a pregnancy, the paternal allele of a child). A calculation of the MFI for the self alleles offers a basis for determining a cutoff value be entered, and the program then determines which epitopes are shared between reactive alleles.

In the clinical setting, the primary purpose of the serum HLA antibody analysis of transplant candidates is to identify potential donors whose mismatched HLA alleles are acceptable. This approach is useful not only for organ transplantation but also for platelet transfusions of allosensitized thrombocytopenic patients. Eurotransplant has incorporated HLAMatchmaker in the Acceptable Mismatch Program to identify donors for highly sensitized patients $(23,24)$.

Epitope specificity analyses might also be useful in desensitization protocols to remove donor-specific antibodies (25). Such protocols are not always uniformly successful but for some patients they may remove some epitope-specific antibodies, thereby opening new windows of opportunity regarding the identification of selected allelic mismatches.

\section{POSTTRANSPLANT MONITORING OF HLA ANTIBODIES}

Many posttransplant studies have shown associations between the appearance of donor-specific antibodies with allograft rejection and failure. Most studies have cases whereby the transplant continues to function quite well in the presence of donor-specific antibodies, and this raises the question which antibodies are clinically significant in transplantation. By definition, such antibodies must be absorbed by the allograft where they recognize epitopes expressed on the vascular endothelium and other tissues and initiate inflammatory processes leading to rejection. Accordingly, testing for circulating HLA antibodies in the presence of a transplant has its limitations, and this becomes apparent with the increased serum reactivity often seen after allograft nephrectomy (26-28), and the identification of donor-specific antibodies in eluates from surgically removed transplants (29-33). The question about epitope specificities of clinically important antibodies can be studied in comparative analyses of pre- and post-allograft nephrectomy sera and better yet by analyzing antibody reactivity patterns of allograft eluates. 


\section{CONTROL OF ANTIBODY RESPONSES TO HLA EPITOPES}

HLA matching at the epitope level also benefits transplant outcome in non-sensitized patients who have no donor-specific HLA antibodies before transplantation. Numerous studies have demonstrated significant correlations between eplet loads of HLA mismatches and the development of donor-specific class I and class II antibodies as well as rejection incidence and allograft outcome (34-48). These findings are clinically useful in two ways. First, for each transplant, the eplet load can be readily determined with special matching programs that can be downloaded from the HLAMatchmaker website. The amount of a mismatched epitope load can be considered as a risk factor during the posttransplant monitoring for antibody-mediated rejection. This information seems also useful in clinical protocols to achieve immunological tolerance (25).

Second, eplet loads can be used to develop new donor selection strategies for non-sensitized recipients especially younger patients. Some transplant programs have already begun to implement this approach (49-51). HLA mismatches with low eplet loads can be expected to improve transplant outcome. Even if the first allograft rejected, retransplant candidates might become less highly sensitized, thereby making it easier to find acceptable mismatches.

The relative immunogenicity of HLA eplets plays an important role in mismatch permissibility of HLA alleles. This can be determined empirically by analyzing the frequencies of antibody responses to mismatched donor eplets which depend on the HLA phenotypes of the recipient and their specificities would be for epitopes defined by eplets or eplet pairs (52). Such studies do not consider the mechanisms of the antibody response which involves the activation of B-cells with epitope-specific Ig-receptors, their subsequent proliferation induced by cytokines from helper T-cells and the differentiation including affinity maturation into antibody-producing plasma cells.

Three recent concepts have begun to address these mechanisms. First, the non-self-self paradigm of HLA epitope immunogenicity is based on the hypothesis that HLA antibodies originate from B-cells with low-avidity, self-HLA epitope-specific Ig-type receptors that can interact with non-self eplets to generate a signal for B-cell activation. Such non-self eplets would be recognized by the specificity-determining CDR-H3, and in the context of the corresponding structural epitope they must be surrounded by self-residues that contact the other CDRs of antibody (53-56).

\section{REFERENCES}

1. Duquesnoy RJ. HLA epitope based matching for transplantation. Transpl Immunol (2014) 31:1-6. doi:10.1016/j.trim.2014.04.004

2. Duquesnoy RJ. Human leukocyte antigen epitope antigenicity and immunogenicity. Curr Opin Organ Transplant (2014) 19:428-35. doi:10.1097/ MOT.0000000000000100

3. Duquesnoy RJ. HLA matching at the epitope level: the way to go. Clin Transpl (2013):441-51.

4. Duquesnoy RJ. Humoral alloimmunity in transplantation: relevance of HLA epitope antigenicity and immunogenicity. Front Immunol (2011) 2:59. doi:10.3389/fimmu.2011.00059
Second, Kosmoliaptsis et al. have proposed that the relative antigenicity of an eplet can be predicted from the physiochemical properties of its amino acid residues (57-59). Accordingly, the electrostatic difference between a non-self eplet and a self-eplet might provide the trigger for B-cell activation (60). Third, activated B-cells need T-cell help for their proliferation and differentiation into antibody-producing plasma cells. The group of Eric Spierings has proposed the so-called PIRCHE-II concept (i.e., Predicted Indirectly ReCognizable HLA Epitopes presented by HLA-DRB1), whereby helper T-cells release cytokines upon recognition of DRB-presented mismatched HLA peptides generated after processing of Ig receptor-allele complexes taken up by activated B-cells $(61,62)$.

These are reasonable theoretical concepts, although no direct experimental methods are available to analyze the very early phases of the antibody response (63). At present, we can only use indirect approaches, such as serum antibody specificity analysis, molecular assessments of matching, and structural analysis of HLA-antigen-antibody complexes, to study the immunogenicity of HLA epitopes. It is important to know a complete repertoire of antibody-verified epitopes. Such investigations could lead to a better understanding of HLA epitope immunogenicity, and how this can be applied to permissible mismatch strategies.

\section{CONCLUSION}

Epitope-based HLA matching has become a new concept in the clinical transplant setting. It relies on HLA typing at the allele level and can be used to identify acceptable mismatches for sensitized patients and to develop permissible mismatch strategies for non-sensitized patients. Our understanding of HLA epitopes is still in progress, and more studies are needed to identify antibody-verified epitopes to be recorded in the HLA Epitope Registry. Also, histocompatibility testing laboratories will have opportunities to sort out complex serum reactivity patterns and interpret technique-dependent epitope specificities of HLA antibodies and their clinical relevance. Sooner or later, there will be new epitope-based HLA-matching approaches with increased benefits to transplant patients.

\section{AUTHOR CONTRIBUTIONS}

The author confirms being the sole contributor of this work and approved it for publication.

5. Terasaki PIE. History of HLA: Ten Recollections. Los Angeles, CA: UCLA Tissue Typing Laboratory (1990).

6. Terasaki PI, McClelland JD. Microdroplet assay of human serum cytotoxins. Nature (1964) 204:998-1000. doi:10.1038/204998b0

7. Yunis EJ, Amos DB. Three closely related genetic systems relevant to transplantation. Proc Natl Acad Sci U S A (1971) 68:3031-3. doi:10.1073/ pnas.68.12.3031

8. Bodmer JG, Pickbourne P, Richards S. In: Bodmer WF, Batchelor JR, Bodmer J, Festenstein H, Morris PJ, editors. Histocompatibility Testing 1977. Copenhagen: Munksgaard (1977).

9. Shaw S, Pollack MS, Payne SM, Johnson AH. HLA-linked B cell alloantigens of a new segregant series: population and family studies of the SB 
antigens. Hum Immunol (1980) 1:177-85. doi:10.1016/0198-8859(80) 90104-4

10. Svejgaard A, Kissmeyer-Nielsen F. Cross-reactive human HL-A isoantibodies. Nature (Lond) (1968) 219:868-9. doi:10.1038/219868a0

11. Colombani J, Colombani M, Degos L, Terrier E, Gaudy Y, Dostot H. Effect of cross reactionson HL-A antigen immunogenicity. Tissue Antigens (1974) 4:136-45. doi:10.1111/j.1399-0039.1974.tb00234.x

12. Rodey GE, Fuller TC. Public epitopes and the antigenic structure of the HLA molecules. Crit Rev Immunol (1987) 7:229-67.

13. Duquesnoy RJ, Filip DJ, Rodey GE, Rimm AA, Aster RH. Successful transfusion of platelets "mismatched" for HLA antigens to alloimmunized thrombocytopenic patients. Am J Hematol (1977) 2:219-26. doi:10.1002/ ajh.2830020303

14. Duquesnoy RJ, Kamoun M, Baxter-Lowe LA, Woodle ES, Bray RA, Claas FHJ, et al. Personal viewpoint: should HLA mismatch acceptability for sensitized transplant candidates be determined at the high-resolution rather than the antigen level? Am J Transplant (2015) 20:1-6. doi:10.1111/ajt.13167

15. Wang J, Yu DT, Fukazawa T, Kellner H, Wen XK, Cheng XK, et al. A monoclonal antibody that recognizes HLA-B27 in the context of peptides. J Immunol (1994) 152:1197-205.

16. Mulder M, Eijsink C, Kester MGD, Franke MEI, Kardol MJ, Heemskerk MHM, et al. Impact of peptides on the recognition of HLA class I molecules by human HLA antibodies. J Immunol (2005) 175:5950-7. doi:10.4049/ jimmunol.175.9.5950

17. Battle R, Turner D, Woodroofe N, Clark B. Identified HLA class I epitopes can indergo conformational induced variation due to changes within HLA bound peptides. Immunol Lett (2014) 161:57-8. doi:10.1016/j.imlet.2014.04.016

18. Tambur AR, Rosati J, Roitberg S, Glotz D, Friedewald JJ, Leventhal JR. Epitope analysis of HLA-DQ antigens: what does the antibody see? Transplantation (2014) 98:157-66. doi:10.1097/TP.0000000000000220

19. Tambur A.HLA-DQ antibodies: are they? Are they relevant? Why so many? Curr Opin Organ Transplant (2016) 21:xx. doi:10.1097/MOT.0000000000000325

20. Duquesnoy RJ, Honger G, Hosli I, Marrari M, Schaub S. Antibody-defined epitopes on HLA-DQ alleles reacting with antibodies induced during pregnancy and the design of a DQ eplet map. Hum Immunol (2016) 77:824-31. doi:10.1016/j.humimm.2016.06.021

21. Duquesnoy RJ, Marrari M, Jelenik L, Zeevi A, Claas FHJ, Mulder A. Structural aspects of HLA class I epitopes reacting with human monoclonal antibodies in Ig-binding, C1q-binding and lymphocytotoxicity assays. Hum Immunol (2013) I74:1271-9. doi:10.1016/j.humimm.2013.05.016

22. Duquesnoy RJ. Epitope-based human leukocyte antigen matching for transplantation. Curr Opin Organ Transplant (2014) 19:418-9. doi:10.1097/ MOT.0000000000000107

23. Claas FHJ, Dankers MK, Oudshoorn M, van Rood JJ, Mulder A, Roelen DL, et al. Differential immunogenicity of HLA mismatches in clinical transplantation. Transpl Immunol (2005) 14:187-91. doi:10.1016/j.trim.2005.03.007

24. Doxiadis IIN, Duquesnoy RJ, Claas FHJ. Extending options for highly sensitized patients to receive a suitable kidney graft. Curr Opin Immunol (2005) 17:536-40. doi:10.1016/j.coi.2005.07.010

25. Duquesnoy RJ. HLA epitopes and tolerance induction protocols (letter to the editor). Am J Transplant (2014) 14:2667. doi:10.1111/ajt.12960

26. Adeyi OE, Girnita A, Awadalla Y, Askar M, Shapiro R, Howe J, et al. Serum analysis after kidney transplant nephrectomy reveals restricted antibody specificity patterns against donor HLA class I antigens. Transpl Immunol (2005) 14:53-62. doi:10.1016/j.trim.2005.01.001

27. Billen EVA, Christiaans MHL, Lee JH, van den Berg-Loonen EM. Donordirected HLA antibodies before and after transplantectomy detected by the luminex single antigen assay. Transplantation (2009) 87:563-9. doi:10.1097/ TP.0b013e3181949e37

28. Marrari M, RJ D. Detection of donor-specific HLA antibodies before and after removal of a rejected kidney transplant. Transpl Immunol (2010) 22:105-9. doi:10.1016/j.trim.2009.12.005

29. Martin L, Guignier F, Mousson C, Rageot D, Justrabo E, Rifle G. Detection of donor-specific anti-HLA antibodies with flow cytometry in eluates and sera from renal transplant recipients with chronic allograft nephropathy. Transplantation (2003) 76:395-400. doi:10.1097/01.TP.0000078895.24606.45

30. Heinemann FM, Roth I, Rebmann V, Arnold ML, Spriewald BM, GrosseWilde H. Characterization of anti-HLA antibodies eluted from explanted renal allografts. Clin Transpl (2006):371-8.
31. Bocrie O, Hussein Aly AA, Guignier F, Funes de la Vega M, Rifle G, Mousson C, et al. Distribution of donor-specific antibodies in the cortex and the medulla of renal transplants with chronic allograft nephropathy. Transpl Immunol (2007) 17:227-9. doi:10.1016/j.trim.2007.01.001

32. Bachelet T, Couzi L, Lepreux S, Legeret M, Pariscoat G, Guidicelli G, et al. Kidney intragraft donor-specific antibodies as determinant of antibody-mediated lesions and poor graft outcome. Am J Transplant (2013) 13:2855-64. doi:10.1111/ajt.12438

33. Milongo D, Kamar N, Del Bello A, Guilbeau-Frugier C, Sallusto F, Esposito L, et al. Allelic and epitopic characterization of intra-kidney-allograft anti-HLA antibodies at allograft nephrectomy. Am J Transplant (2016). doi:10.1111/ ajt.13958

34. Dankers MKA, Witvliet MD, Roelen DL, De Lange P, Korfage N, Persijn GG, et al. The number of amino acid triplet differences between patient and donor is predictive for the antibody reactivity against mismatched HLA antigens. Transplantation (2004) 77:1236-9. doi:10.1097/01.TP.0000120385. 03278.28

35. Goodman RS, Taylor CJ, O’Rourke CM, Lynch A, Bradley JA, Key T. Utility of HLAMatchmaker and single-antigen HLA-antibody detection beads for identification of acceptable mismatches in highly sensitized patients awaiting kidney transplantation. Transplantation (2006) 81:1331-6. doi:10.1097/01. tp.0000205202.56915.f5

36. Kosmoliaptsis V, Bradley JA, Sharples LD, Chaudhry A, Key T, Goodman RS, et al. Predicting the immunogenicity of human leukocyte antigen class I alloantigens using structural epitope analysis determined by HLAMatchmaker. Transplantation (2008) 85:1817-25. doi:10.1097/TP.0b013e31817441d6

37. Duquesnoy RJ, Awadalla Y, Lomago J, Jelinek L, Howe J, Zern D, et al. Retransplant candidates have donor-specific antibodies that react with structurally defined HLA-DR,DQ,DP epitopes. Transpl Immunol (2008) 18:352-60. doi:10.1016/j.trim.2007.10.001

38. Kosmoliaptsis V, Chaudhry AN, Sharples LD, Halsall DJ, Dafforn TR, Bradley JA, et al. Predicting HLA class I alloantigen immunogenicity from the number and physiochemical properties of amino acid polymorphisms. Transplantation (2009) 88:791-8. doi:10.1097/TP.0b013e3181b4a9ff

39. Duquesnoy RJ, Marrari M. Detection of antibodies against HLA-C epitopes in patients with rejected kidney transplants. Transpl Immunol (2011) 24:164-71. doi:10.1016/j.trim.2010.12.003

40. Honger G, Fornaro I, Granado C, Tiercy JM, Hosli I, Schaub S. Frequency and determinants of pregnancy-induced child-specific sensitization. Am J Transplant (2013) 13:746-53. doi:10.1111/ajt.12048

41. Wiebe C, Nickerson P. Posttransplant monitoring of de novo human leukocyte antigen donor-specific antibodies in kidney transplantation. Curr Opin Organ Transplant (2013) 18:470-7. doi:10.1097/MOT.0b013e3283626149

42. Wiebe C, Nickerson P. Acceptable mismatching at the class II epitope level: the Canadian experience. Curr Opin Organ Transplant (2014) 19:442-6. doi:10.1097/MOT.0000000000000104

43. Wiebe C, Nevins TE, Robiner WN, Thomas W, Matas AJ, Nickerson PW. The synergistic effect of class II HLA epitope mismatch and nonadherence on acute rejection and graft survival. Am J Transplant (2015) 15:2197-202. doi:10.1111/ajt.13341

44. Filippone EJ, Farber JL. Humoral immunity in renal transplantation: epitopes, Cw and DP, and complement activating capability - an update. Clin Transplant (2015) 29:279-87. doi:10.1111/ctr.12524

45. Sapir-Pichhadze R, Tinckam K, Quach K, Logan AG, Laupacis A, John R, et al. HLA-DR and -DQ eplet mismatches and transplant glomerulopathy: a nested case-control study. Am J Transplant (2015) 15:137. doi:10.1111/ajt.12968

46. Singh P, Filippone E, Colombe B, Shah A, Zhan T, Harach M, et al. Sensitization trends after renal allograft failure: the role of DQ eplet mismatches in becoming highly sensitized. Clin Transplant (2016) 30:71-80. doi:10.1111/ctr.12663

47. Sullivan P, Warner P, Kemna M, Albers E, Law S, Weiss N, et al. HLA molecular epitope mismatching and long-term graft loss in pediatric heart transplant recipients. J Heart Lung Transplant (2016) 34:950-7. doi:10.1016/j. healun.2014.12.017

48. Walton D, Hiho S, Cantwell LS, Diviney M, Wright S, Snell G, et al. HLA matching at the eplet level protects against chronic lung allograft dysfunction. Am J Transplant (2016) 20:1-9. doi:10.1111/ajt.13798

49. Bryan C, Chadha V, Warad B. Donor selection in pediatric kidney transplantation using DR and DQ eplet mismatching: a new histocompatibility paradigm. Pediatr Transplant (2016) 20. (in press). 
50. Kausman JY, Walker AM, Cantwell LS, Quinlan C, Sypek MP, Ierino FL. Application of an epitope-based allocation system in paediatric kidney transplantation. Pediatr Transplant (2016) 20. (in press).

51. Duquesnoy RJ. The eplet load concept in clinical transplantation (editorial). Pediatr Transplant (2016) 20. (in press).

52. Duquesnoy RJ, Claas FHJ. Progress report of 14th international histocompatibility workshop project on the structural basis of HLA compatibility. Tissue Antigens (2007) 69(Suppl 1):180-4. doi:10.1111/j.1399-0039.2006. 00766.x

53. Duquesnoy RJ, Marrari M, Mulder A. Usefulness of the nonself-self algorithm of HLA epitope immunogenicity in the specificity analysis of monospecific antibodies induced during pregnancy. Front Immunol (2015) 6:160. doi:10.3389/fimmu.2015.00180

54. Hahn A, Bravo-Egana V, Jackstadt J, Conti D, Duquesnoy RJ. HLA-A2 reactive antibodies in a patient who types as HLA-A2: the importance of high 3 resolution typing and epitope-based antibody analysis. Transpl Immunol (2015) 32:141-3. doi:10.1016/j.trim.2015.04.001

55. Resse M, Paolillo R, Minucci B, Moccia G, Napoli C. Antibody-reactive class I epitopes defined by pairs of mismatched eplets and self-eplets. Tissue Antigens (2015) 86:368-72. doi:10.1111/tan.12652

56. Daniëls L, Emonds M, Bosmans J, Marrari M, Duquesnoy RJ. Epitope analysis of DQB antibody reactivity in sera from a transplant candidate sensitized during pregnancy. Transpl Immunol (2016). doi:10.1016/j.trim.2016.07.004

57. Kosmoliaptsis V, Sharples LD, Chaudhry AN, Halsall DJ, Bradley JA, Taylor CJ. Predicting HLA class II alloantigen immunogenicity from the number and physiochemical properties of amino acid polymorphisms. Transplantation (2011) 91:183-90. doi:10.1097/TP.0b013e3181ffff99

58. Mallon DH, Bradley JA, Taylor CJ, Kosmoliaptsis V. Structural and electrostatic analysis of HLA B-cell epitopes: inference on immunogenicity and prediction of humoral alloresponses. Curr Opin Organ Transplant (2014) 19:420-7. doi:10.1097/MOT.0000000000000108
59. Mallon DH, Bradley JA, Winn PJ, Taylor CJ, Kosmoliaptsis V. Threedimensional structural modelling and calculation of electrostatic potentials of HLA Bw4 and Bw6 epitopes to explain the molecular basis for alloantibody binding: toward predicting HLA antigenicity and immunogenicity. Transplantation (2015) 99:385-90. doi:10.1097/TP.0000000000000546

60. McCaughan J, Turner D, Battle R. Electrostatic potential change in a paired epitope: a novel explanation for Bw4 antibodies in patients with B13(Bw4) antigens. Transplantation (2016) 100:xx. doi:10.1097/TP.0000000000001193

61. Otten HG, Calis JJA, Kesmir C, van Zuilen AD, Spierings E. Predicted indirectly recognizable HLA epitopes presented by HLA-DR correlate with the de novo development of donor-specific HLA IgG antibodies after kidney transplantation. Hum Immunol (2013) 74:290-6. doi:10.1016/j.humimm. 2012.12.004

62. Geneugelijk K, Honger G, van Deutekom H, Thus K, Kesmir C, Hosli I, et al. Predicted indirectly recognizable HLA epitopes presented by HLA-DRB1 (PIRCHE-II) are related to HLA-antibody formation during pregnancy. Am J Transplant (2015) 15:3112-22. doi:10.1111/ajt.13508

63. Duquesnoy RJ. The antibody response to a HLA mismatch: putting together the pieces of a puzzle. Am J Transplant (2015) 15:3019-20. doi:10.1111/ ajt.13510

Conflict of Interest Statement: The author declares that the research was conducted in the absence of any commercial or financial relationships that could be construed as a potential conflict of interest.

Copyright (๑) 2016 Duquesnoy. This is an open-access article distributed under the terms of the Creative Commons Attribution License (CC BY). The use, distribution or reproduction in other forums is permitted, provided the original author(s) or licensor are credited and that the original publication in this journal is cited, in accordance with accepted academic practice. No use, distribution or reproduction is permitted which does not comply with these terms. 